\title{
Investigating the interpretability of fetal status assessment using antepartum cardiotocographic records
}

Liting Huang ${ }^{1,2}$, Zhiying Jiang ${ }^{2}$, Ruichu Cai ${ }^{1}$, Li Li $i^{3,5}$, Qinqun Chen ${ }^{2}$, Jiaming Hong ${ }^{2}$, Zhifeng Hao ${ }^{4}$ and Hang $\mathrm{Wei}^{2^{*}}$ (D)

\begin{abstract}
Background: Cardiotocography (CTG) interpretation plays a critical role in prenatal fetal monitoring. However, the interpretation of fetal status assessment using CTG is mainly confined to clinical research. To the best of our knowledge, there is no study on data analysis of CTG records to explore the causal relationships between the important CTG features and fetal status evaluation.
\end{abstract}

Methods: For analyses, 2126 cardiotocograms were automatically processed and the respective diagnostic features measured by the Sisporto program. In this paper, we aim to explore the causal relationships between the important CTG features and fetal status evaluation. First, we utilized data visualization and Spearman correlation analysis to explore the relationship among CTG features and their importance on fetal status assessment. Second, we proposed a forward-stepwise-selection association rule analysis (ARA) to supplement the fetal status assessment rules based on sparse pathological cases. Third, we established structural equation models (SEMs) to investigate the latent causal factors and their causal coefficients to fetal status assessment.

Results: Data visualization and the Spearman correlation analysis found that thirteen CTG features were relevant to the fetal state evaluation. The forward-stepwise-selection ARA further validated and complemented the CTG interpretation rules in the fetal monitoring guidelines. The measurement models validated the five latent variables, which were baseline category (BCat), variability category (VCat), acceleration category (ACat), deceleration category (DCat) and uterine contraction category (UCat) based on fetal monitoring knowledge and the above analyses. Furthermore, the interpretable models discovered the cause factors of fetal status assessment and their causal coefficients to fetal status assessment. For instance, VCat could predict BCat, and UCat could predict DCat as well. ACat, BCat and DCat directly affected fetal status assessment, where ACat was the important causal factor.

Conclusions: The analyses revealed the interpretation rules and discovered the causal factors and their causal coefficients for fetal status assessment. Moreover, the results are consistent with the computerized fetal monitoring and clinical knowledge. Our approaches are conducive to evidence-based medical research and realizing intelligent fetal monitoring.

Keywords: Fetal monitoring, Cardiotocography, Forward-stepwise-selection association rule analysis, Structural equation model

\footnotetext{
${ }^{*}$ Correspondence: crwei@gzucm.edu.cn

${ }^{2}$ School of Medical Information Engineering, Guangzhou University

of Chinese Medicine, Waihuandong Road, Guangzhou, China

Full list of author information is available at the end of the article
} original author(s) and the source, provide a link to the Creative Commons licence, and indicate if changes were made. The images or other third party material in this article are included in the article's Creative Commons licence, unless indicated otherwise in a credit line to the material. If material is not included in the article's Creative Commons licence and your intended use is not permitted by statutory regulation or exceeds the permitted use, you will need to obtain permission directly from the copyright holder. To view a copy of this licence, visit http://creativecommons.org/licenses/by/4.0/. The Creative Commons Public Domain Dedication waiver (http://creativeco mmons.org/publicdomain/zero/1.0/) applies to the data made available in this article, unless otherwise stated in a credit line to the data. 


\section{Background}

Cardiotocography (CTG) was widely introduced into antenatal fetal monitoring in the late 1960s and is still widely used due to its low cost, ease of operation and non-invasiveness [1]. Through recording fetal heart rate (FHR) and uterine contraction (UC) signals in non-stress testing (NST) [2, 3], it helps early diagnosis of pathological cases, such as congenital heart defects, fetal distress and hypoxia, which can timely prevent irreversible damage to the fetus [4].

The mainstream fetal monitoring guidelines include Society of Obstetricians and Gynaecologists of Canada (SOGC) [5], American College of Obstetricians and Gynecologists (ACOG), National Institute for Health and Care Excellence (NICE), International Federation of Obstetrics and Gynecology (FIGO) [6, 7], and Chinese expert consensus [8]. Obstetricians usually assess fetal health status by visually interpreting the morphological CTG features, including baseline, variation, deceleration and so on, and then apply them to the corresponding fetal monitoring guidelines. However, visual analysis of CTG records by guidelines usually leads to high intra-observer and inter-observer disagreement among obstetricians [9]. Thus some researchers focused on developing the automated CTG analysis software to extract CTG features from FHR and UC signals. For instance, SYSTEM 8000 was developed for antenatal FHR analysis [10]. Openaccess software CTG-OAS was introduced for the automatic analysis of FHR signals [11]. CTG Analyzer was proposed as a graphical tool for automatic and objective analysis of CTG tracings [12]. The Sisporto program was developed to analyze FHR and UC signals automatically and used a relatively complex algorithm to estimate the mean FHR during periods of fetal rest without reducing signals, which can evaluate the closest variability of beatto-beat $[13,14]$.

With the computerized CTG analysis technology, some researchers combined CTG features with clinical theoretical studies. They identified biomarkers on CTG records that helped to diagnosis of pathological cases, like distress, growth restriction and cardiac arrhythmia. For example, fetal distress was considered to be associated with variable deceleration and hyperdynamic uteri [15]. Meanwhile, some maternal diseases could be judged by prenatal CTG features [4].

In recent decades, many researchers applied machine learning methods to classify CTG data for fetal status assessment. The study [16] evaluated the classification performance of eight different machine learning methods on prenatal CTG data, which showed the Bagging with Random Forest achieved better results with an accuracy of $99.02 \%$. Yang et al. used hybrid principal component analysis (PCA) and adaptive enhancement (AdaBoost)
Table 1 Attribute Information about the CTG dataset

\begin{tabular}{ll}
\hline Symbol & Feature description \\
\hline LB & FHR baseline (beats per minute) \\
AC & Number of accelerations per second \\
FM & Number of fetal movements per second \\
UC & Number of uterine contractions per second \\
DL & Number of light decelerations per second \\
DS & Number of severe decelerations per second \\
DP & Number of prolonged decelerations per second \\
ASTV & Percentage of time with abnormal short term variability \\
MSTV & Mean value of short term variability \\
ALTV & Percentage of time with abnormal long term variability \\
MLTV & Mean value of long term variability \\
Width & Width of FHR histogram \\
Min & Minimum of FHR histogram \\
Max & Maximum of FHR histogram \\
Nmax & Number of histogram peaks \\
Nzeros & Number of histogram zeros \\
Mode & Histogram mode \\
Mean & Histogram mean \\
Median & Histogram median \\
Variance & Histogram variance \\
Tendency & Histogram tendency \\
NSP & Fetal state class code ( $N=1 ; \mathrm{S}=2 ; \mathrm{P}=3$ ) \\
\hline
\end{tabular}

to successfully classify CTG data and assessed fetal status with an accuracy of 98.6\% [17]. In literature [18], Vinayaka Nagendra used RF and support vector machine (SVM) to perform a three-categories study of the fetal status, and the results showed that the accuracy was higher than $96 \%$. Zhao et al. used statistical test (ST), the area under the curve (AUC) and PCA to select features, and applied three representative machine learning algorithms, decision tree (DT), the SVM and AdaBoost to classify the fetal status as two categories [19].

The above studies focused on the relationships between CTG features and fetal status or diseases in clinical research, or directly established evaluation models by machine learning methods. However, they did not pay attention to the interpretability of fetal status assessment. Consequently, it is significant to investigate the interpretability of fetal status assessment using cardiotocographic records. In this study, data visualization, Spearman correlation analysis, forward-stepwise-selection ARA, and SEM models were implemented to achieve the following goals: (1) identifying important CTG features of great impact on fetal status; (2) exploring the association rules between CTG features and fetal status to supplement fetal monitoring guidelines; (3) exploring the causal relationship among latent variables and the causal relationship of latent variables to fetal status. 


\section{Methods}

\section{Data and analysis tools}

For analyses, we selected the cardiotocography (CTG) dataset from the public available UCI Machine Learning Repository [20], which has been validated by numerous machine learning-based fetal state assessment researches with the accuracy of higher than 95\% [16-19]. In the dataset, 2126 CTG signals with 29-42 gestational weeks were automatically processed and the respective 21 diagnostic features were measured by the SisPorto2.0 program undergoing a multicenter validation study (http:// sisporto.med.up.pt) [13]. The FHR feature computation was based on consensual guidelines, such as the International Federation of Gynecology and Obstetrics (FIGO) and the National Institutes of Health. It involved 11 steps in successive order, including FHR spike removal, filtering of uterine contraction signals, detection of uterine contractions, and so on. The attribute information of 21 diagnostic features can be found in Table 1. The CTGs were also interpreted by three expert obstetricians according to the FIGO guideline shown in Table 2 and a consensus classification label concerning fetal state (NSP: normal, suspicious and pathology) assigned to each of them. In all, there are 1655, 295 and 176 instances for each category respectively. Figure 1 shows that the proportion of the categories was imbalanced. The normal, suspicious and pathology cases accounted for $78 \%, 14 \%$ and $8 \%$, respectively.
Data visualization, Spearman correlation analysis and forward-stepwise-selection ARA were implemented using programming language Python3.8 and the compilers Pycharm Professional 2020.3.2. The measurement model and the structural equation model were analyzed by AMOS 24.0 software.

\section{Correlation and significance analysis}

The relationships among CTG features and those between CTG features and fetal status (NSP) were discussed by Spearman's rank correlation coefficient, which measured the degree of correlation between grading sequencing variables.

\section{Forward-stepwise-selection ARA}

Normally, pathology cases are seriously scarce in clinical practice. While the traditional Apriori algorithms $[21,22]$ in ARA can not achieve the infrequent association rules and the improved method based on increasing the weight of minority would produce biased results as well $[6,23]$. Therefore we designed an algorithm of forward-stepwiseselection association rule to maximize the accuracy of association rules. The CTG features were firstly discretized by fetal status distribution and the basic flow was shown in Algorithm 1.

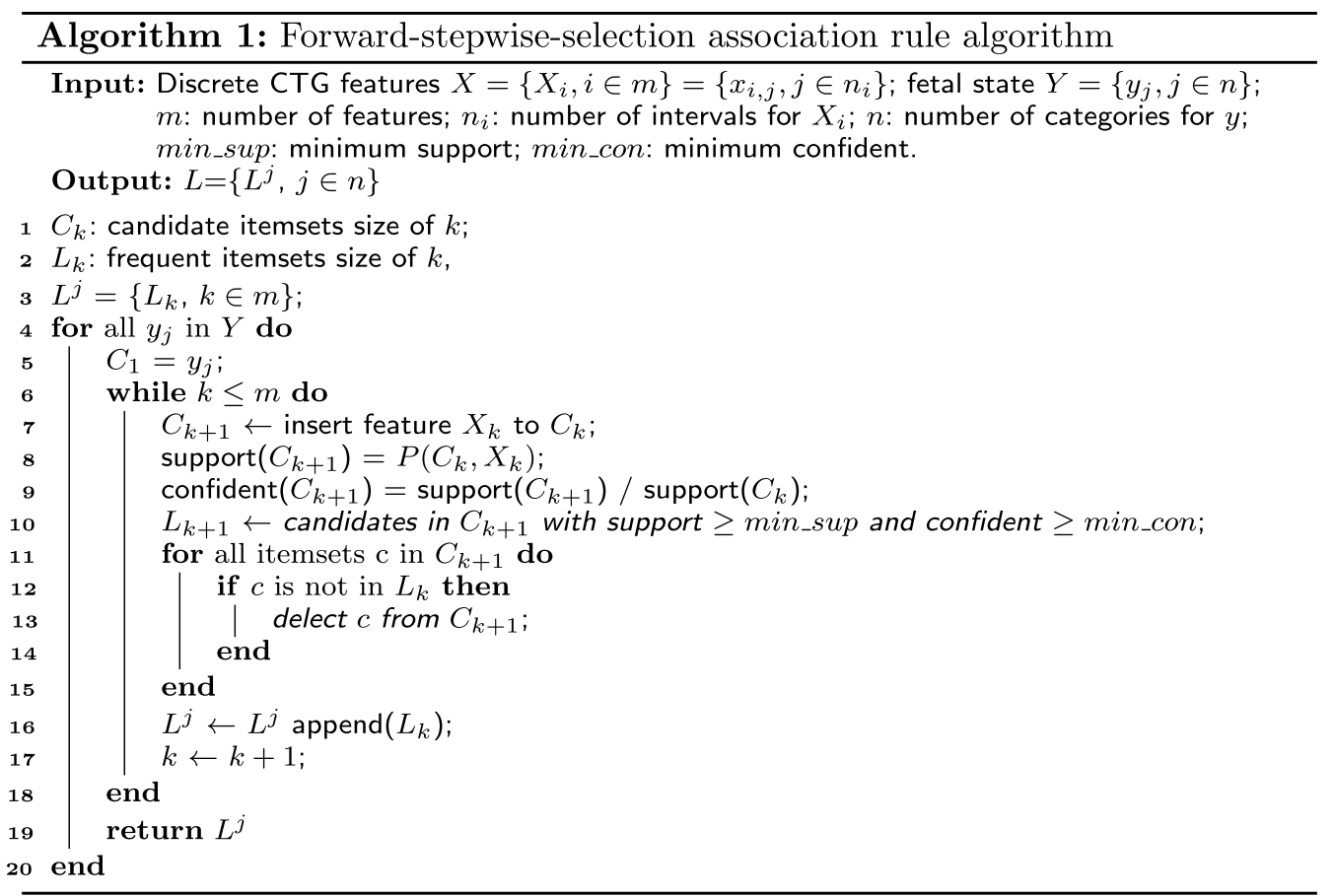


Table 2 The definition of three categories of fetal status (normal, suspicious and pathology) according to the FIGO guidelines

\begin{tabular}{llll}
\hline & Normal & Suspicious & Pathology \\
\hline Baseline & Between 110 and $150 \mathrm{bpm}$ & $\begin{array}{l}\text { Between } 100 \text { and } 110 \mathrm{bpm} \text { or between } 150 \text { and } 170 \\
\text { bpm }\end{array}$ & $\begin{array}{l}\text { Below } 100 \text { or above } 170 \mathrm{bpm} \\
\text { Variablity }\end{array}$ \\
Others & Between 5 and $25 \mathrm{bpm}$ & $\begin{array}{l}\text { Amplitude of variability between } 5 \text { and } 10 \mathrm{bpm} \text { for } \\
\text { more than } 40 \mathrm{~s}\end{array}$ & $\begin{array}{l}\text { Persistence of heart rate variability of less than } 5 \text { bpm } \\
\text { Increased variability above } 25 \mathrm{bpm} \text {; Variable decelera- } \\
\text { tions }\end{array}$ \\
& $\begin{array}{l}\text { Severe variable decelerations or severe repetitive early } \\
\text { decelerations; Prolonged decelerations; Late decelera- } \\
\text { tions: the most ominous trace is a steady baseline } \\
\text { without baseline variability and with small decelera- } \\
\text { tions after each contraction; A sinusoidal pattern }\end{array}$ \\
\hline
\end{tabular}

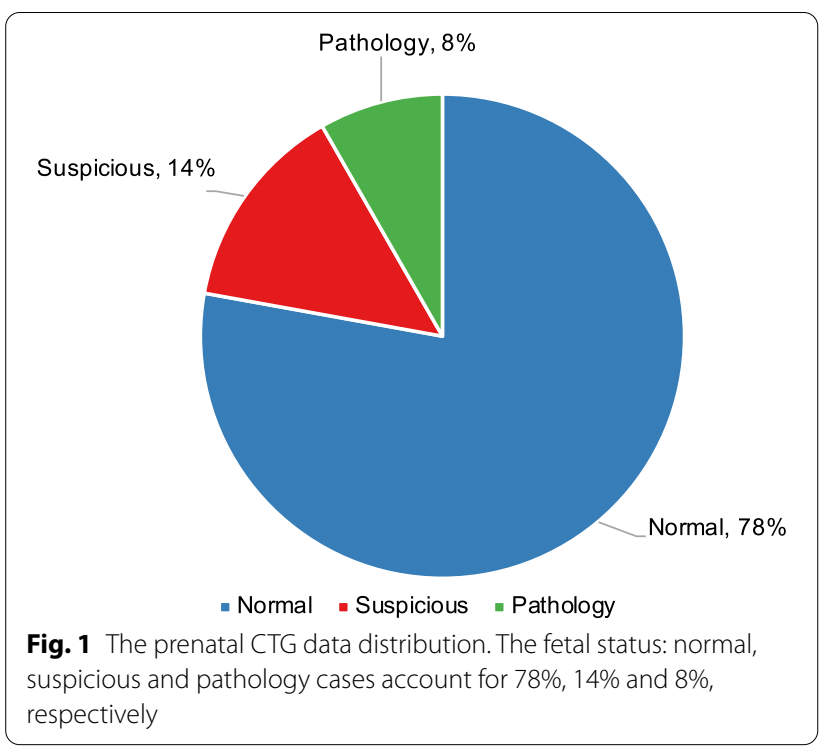

\section{SEMs for interpreting prenatal fetal monitoring}

Structural equation modeling is referred to as covariance structural analysis that combines confirmatory factor analysis (CFA) and regression or path analysis. First, the latent variables that can be represented by their observed variables were assessed by estimating the measurement models. If the measurement models satisfied the fitting indices requirements, the final measurement model would be adopted to investigate the impact of latent variables. Furthermore, structural equation models (SEMs) were introduced to study the relationship between the observed variables and the latent variables, the causation among latent variables, and the mechanism of the influence of each latent variable on fetal status. The theoretical model of SEMs is shown in Fig. 2. The relationships between observation variables and latent variables are expressed as Eqs. (1) and (2).

$$
\begin{aligned}
& x=\Lambda_{x} \xi+\delta \\
& y=\Lambda_{y} \eta+\epsilon
\end{aligned}
$$

where $x$ and $y$ are prenatal fetal monitoring features, $\xi$ and $\eta$ are latent variables, $\delta$ and $\epsilon$ are unique factor vectors. The simple form of the structural equation model is a multiple regression model with only one dependent

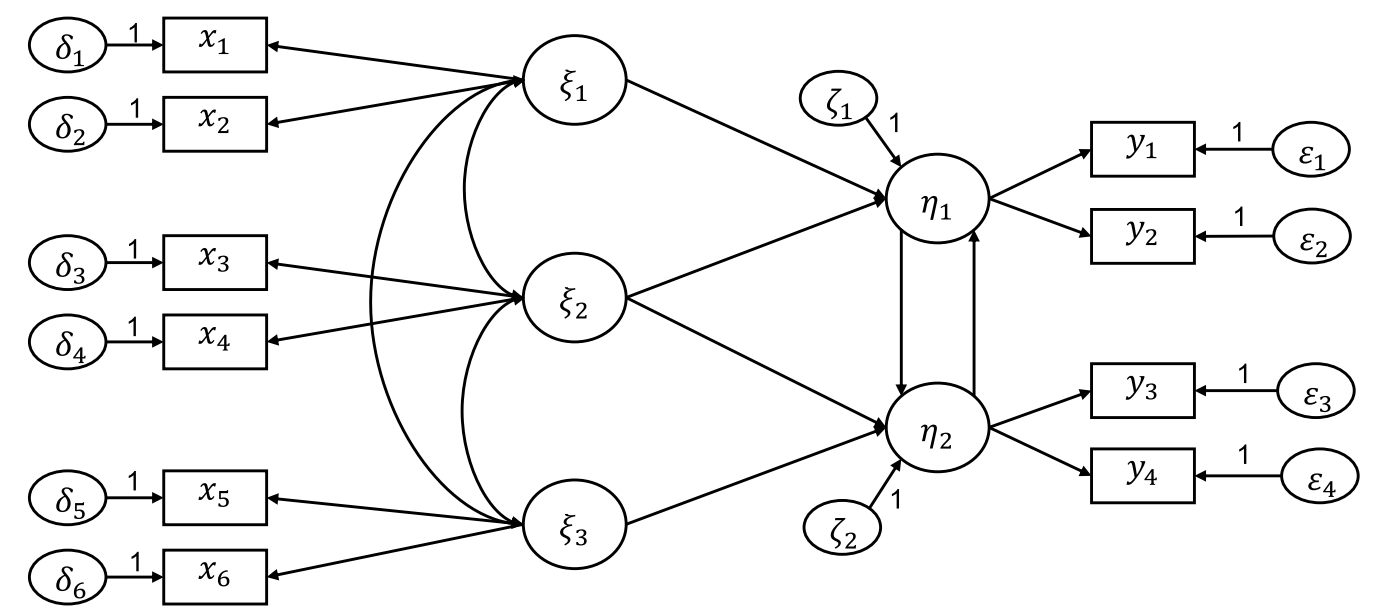

Fig. 2 An hypothetical example of a structural equation model. The single-headed arrow represents causal relationship, the double-headed arrow indicates unresolved causality 


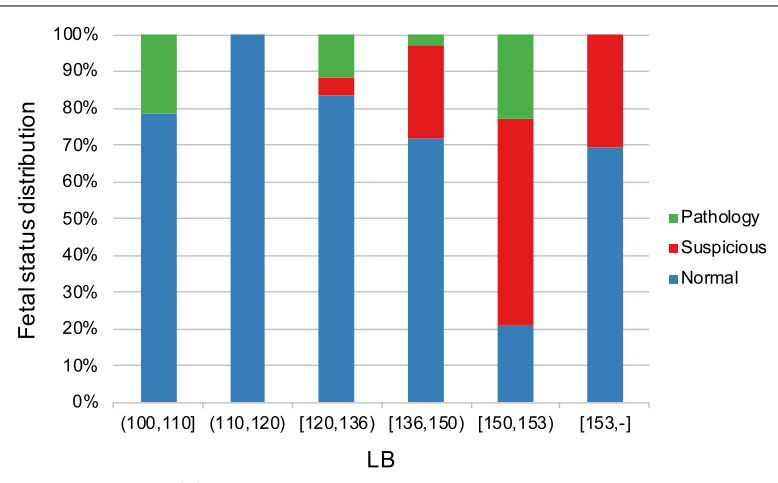

(a) The distribution of LB to fetal status

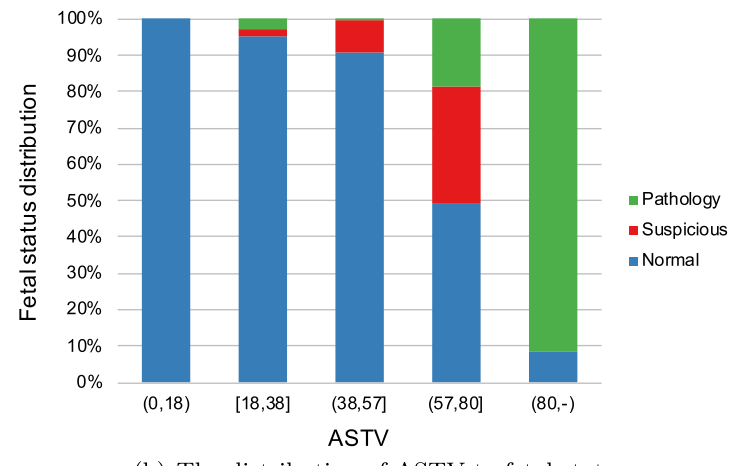

(b) The distribution of ASTV to fetal status

Fig. 3 Distribution of fetal status to features proportion

variable. The Eq. (3) is as follows, where $\eta$ is determined by y and $\epsilon$.

$$
\eta=\boldsymbol{\beta} \eta+\boldsymbol{\Gamma} \xi+\zeta
$$

\section{Results}

\section{Exploration of the important CTG features}

The proportion of the fetal status distribution within a certain range for each CTG feature was visualized by percentage stacked bar charts. The experimental data visualization revealed the following findings. When the values of AC, UC and MSTV increased, the fetal status tended to be normal. In contrast, when the values of ASTV, ALTV, DP and LB increased, the fetus was more likely to be pathological. Figure 3 illustrates two instances. Firstly, the baseline (LB) values in the normal cases were mostly at the interval of 111-120 bpm, while the numbers of suspicious and pathology cases increased with baseline values. Secondly, the proportion of pathology cases increased with ASTV, whereas that of normal reversed; when ASTV value ranged from 0 to 18, the proportion of normal category reached $100 \%$; as the ASTV value exceeded 80 , the proportion of pathological cases increased significantly. Overall, twelve CTG features symbolized as AC, UC, LB, FM, DP, ASTV, ALTV, MLTV, Mode, Mean, Min, and Median were closely related to the fetal status.

Figure 4 is a thermal map calculated by Spearman's coefficient, showing highly correlated feature groups and important features associated with fetal status. Ten features with high correlation among the 21 features were divided into two groups listed in Table 3. In addition, the CTG features correlated with pathological fetal were found to be MSTV, MLTV and ASTV $(R=0.5,0.39$ and 0.34 , respectively); the features associated with normal fetal were AC, ALTV and UC $(R=-0.46,-0.29$ and -0.26 , respectively).

\section{Supplement of the fetal monitoring guidelines}

We explored the relationship between CTG features and fetal status using forward-stepwise-selection association rules. The results showed that MSTV, AC, Mean, ASTV and Min were the decisive features to normal fetal status. When the values of those were within certain ranges, the confidence values for normal fetal state attained $100 \%$. On the other hand, the key features to pathological fetal status were DP, DS, Variance, and Mean. When the values of those ranged in certain intervals, the confidence values of fetal status for pathology reached $100 \%$. In summary, the CTG interpretation rules excavated by the proposed ARA verified and supplemented the fetal monitoring guidelines displayed in Table 4.

\section{The explanation mechanism of prenatal CTG feature on fetal status The result of measurement model assumptions}

Combined with FIGO guidelines and clinical knowledge $[24,25]$, seventeen features with great influence on fetal status were obtained through data visualization, Spearman correlation analysis and forward-stepwise-selection association rules. On this basis, seventeen features were divided into five categories named baseline category (BCat), variability category (VCat), acceleration category (ACat), deceleration category (DCat) and uterine contraction category (UCat). And a measurement model (Fig. 5) assuming all features could be explained by their latent variables (categories) was established. The measurement model using the oblique confirmatory factor analysis method not only verified whether the latent variables we hypothesized had meaningful interpretation to the observed variables, but also explored the correlation among the five latent variables in this study. In Table 5, except for the MLTV, the other 16 features were all statistically significant $(P<0.001)$, confirming our hypotheses that these five latent variables were meaningful in explaining their respective observed variables [26]. 


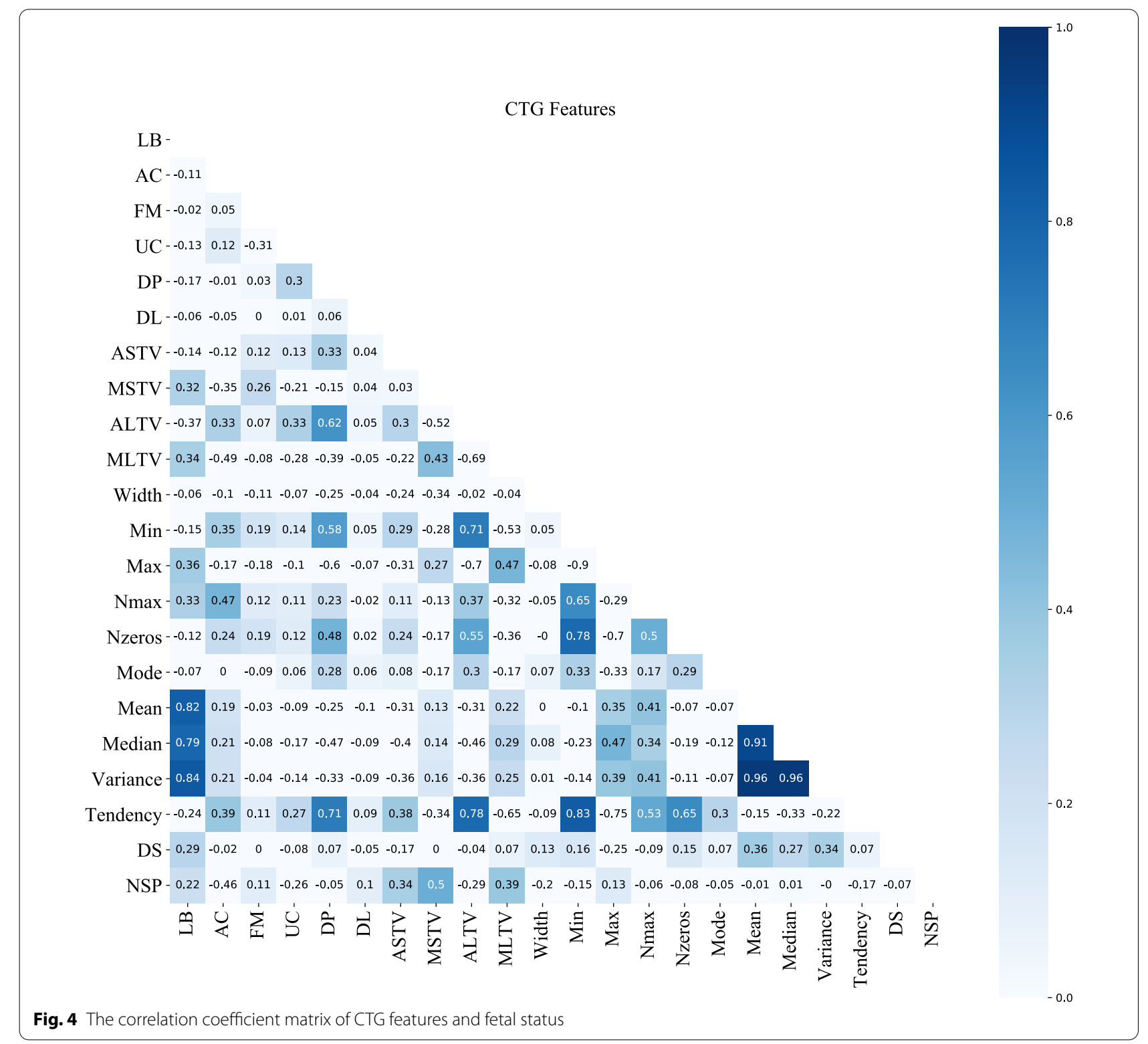

Table 3 Grouping of ten highly correlated CTG features

\begin{tabular}{ll}
\hline Group & Correlated feature \\
\hline 1 & LB, Mode, Median, Mean \\
2 & Width,Variance, Nmax, MSTV, Max, DL
\end{tabular}

\section{Interpretability of prenatal fetal status assessment}

The purpose of this part was to derive the mechanism of prenatal CTG features and fetal status. Structural equation modeling was proved to be an effective tool to recognize the different impacts of five latent variables on fetal status evaluation. After the model fitting adjustment [27, 28 ], the structure model was obtained as shown in Fig. 6.

Table 6 lists the results of the structural equation model. All the hypothesis relationships, including the interpretation of latent variables on observed variables, the causality among latent variables, and the influence of latent variables on fetal status were statistically significant $(P<0.05)[29,30]$. Table 6 and Fig. 6 show that ACat, BCat, and DCat had direct effects on fetal status with the standardized path coefficients of $-0.665,-0.240$, and $0.173(P<0.05)$, respectively. It indicated that the fetal status changes from normal to pathology as DCat increases, whereas it has opposite change as ACat and 
Table 4 Supplement to the fetal monitoring guidelines

\begin{tabular}{lll}
\hline Fetal status & FIGO & Supplement \\
\hline Normal & MSTV: Average STV; $;$ ASTV: percentage of points with STV & AC: $[0.0048,0.005]$ times per \\
& second; MSTV: [0.6, 1.5); Mean: \\
& {$[125.175,138.23) ;$ Min: $[86,108) ;$} & ASTV: $[30,44)$ \\
& DS: lasting at least 15 s and with amplitude exceeding 15 bpm; DP: lasting & Mode: range in [60, 99); Variance: \\
Pathology & $>165 ;$ DS: $>0.00094$ times per \\
& & second; DP: $>0.0028$ times per \\
& second & \\
\hline
\end{tabular}

${ }^{\text {a }}$ STV is identified whenever the difference between two adjacent FHR signals is less than $1 \mathrm{bpm}$

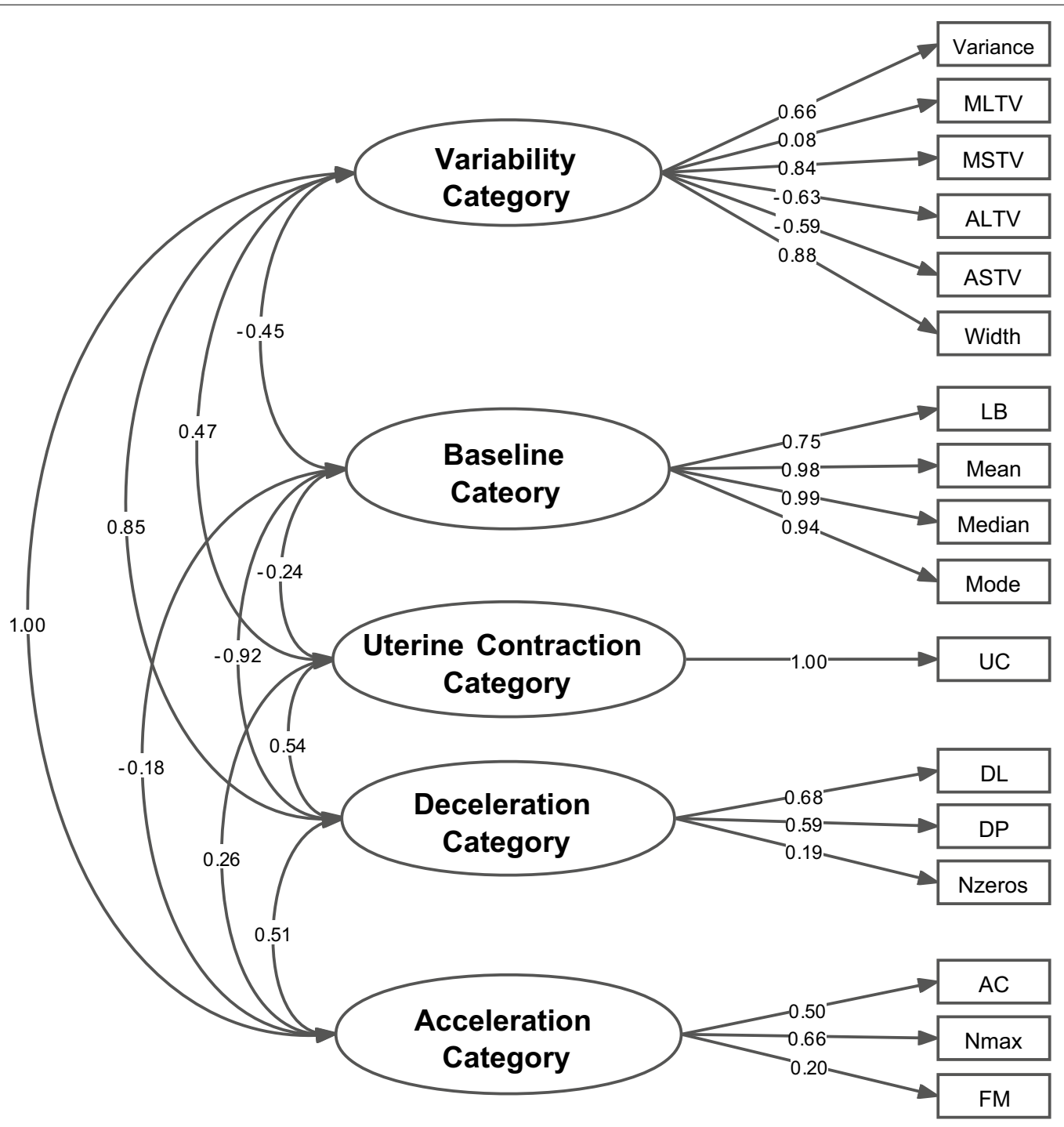

Fig. 5 The measurement model for five latent constructs and seventeen observed variables. Note: The values on an arrow are standard path coefficients (also known as regression coefficients); the values on double arrows indicate the correlationships among the five latent variables 
Table 5 Standardized regression weighting coefficients and parameter estimates for observed variables to latent variables

\begin{tabular}{|c|c|c|c|}
\hline Hyoithesized path & Estimate $^{a}$ & C.R. ${ }^{b}$ & $P$ values ${ }^{c}$ \\
\hline Variance $\longleftarrow$ VCat & 0.662 & & \\
\hline MLTV «VCat & 0.084 & 2.296 & 0.282 \\
\hline MSTV $\longleftarrow$ VCat & 0.837 & 20.662 & $* * *$ \\
\hline ALTV $\longleftarrow$ VCat & -0.632 & -16.244 & $* * *$ \\
\hline ASTV $\longleftarrow$ VCat & -0.589 & -15.248 & $* * *$ \\
\hline Width $\longleftarrow$ VCat & 0.880 & 21.493 & $* * *$ \\
\hline $\mathrm{LB} \longleftarrow \mathrm{BCat}$ & 0.748 & & \\
\hline Mean $\longleftarrow$ BCat & 0.977 & 30.763 & $* * *$ \\
\hline Median $\longleftarrow$ BCat & 0.990 & 31.232 & $* * *$ \\
\hline Mode $\longleftarrow$ BCat & 0.937 & 29.210 & $* * *$ \\
\hline $\mathrm{DL} \longleftarrow$ DCat & 0.680 & 16.818 & $* * *$ \\
\hline $\mathrm{DP} \longleftarrow$ DCat & 0.586 & & \\
\hline Nzeros « DCat & 0.191 & 5.561 & $* * *$ \\
\hline $\mathrm{AC} \longleftarrow \mathrm{ACat}$ & 0.497 & & \\
\hline $\mathrm{Nmax} \longleftarrow$ ACat & 0.660 & 14.527 & $* * *$ \\
\hline $\mathrm{FM} \longleftarrow$ ACat & 0.201 & 5.891 & $* * *$ \\
\hline$U C \longleftarrow$ UCat & 1.000 & & \\
\hline VCat $\longleftrightarrow$ BCat & -0.447 & -9.549 & $* * *$ \\
\hline DCat $\longleftrightarrow$ ACat & 0.51 & 7.004 & $* * *$ \\
\hline VCat $\longleftrightarrow$ DCat & 0.848 & 11.498 & $* * *$ \\
\hline VCat $\longleftrightarrow$ ACat & 1.00 & 11.556 & $* * *$ \\
\hline BCat $\longleftrightarrow$ DCat & -0.921 & -12.737 & $* * *$ \\
\hline BCat $\longleftrightarrow$ ACat & -0.178 & -3.547 & $* * *$ \\
\hline UCat $\longleftrightarrow$ BCat & -0.241 & -6.438 & $* * *$ \\
\hline UCat $\longleftrightarrow$ DCat & 0.541 & 10.042 & $* * *$ \\
\hline UCat $\longleftrightarrow$ ACat & 0.26 & 5.113 & $* * *$ \\
\hline UCat $\longleftrightarrow$ VCat & 0.467 & 10.366 & $* * *$ \\
\hline
\end{tabular}

***Significant at $P<0.001$

${ }^{\text {a }}$ Standardized regression coefficients of estimate

${ }^{b}$ Critical ratio (C.R.) is the $t$ value of T-test

$c P>0.05$ Implied that the hypothetical pathways were false

BCat increase. In addition, UCat and VCat were high correlated $(r=-0.925)$ and had an indirect impact on the fetal status by affecting DCat and BCat with the standardized indirect effect of 0.95 and 0.53 , respectively.

\section{Discussion}

Data visualization and Spearman correlation analysis found some important features to fetal state. Visual analysis was conducted from the perspective of data distribution and obtained twelve important features affecting fetal status: AC, UC, LB, FM, DP, ASTV, ALTV, MLTV, Mode, Mean, Min, and Median. It was also found that features related to pathological fetal were MSTV, MLTV, and ASTV, and features related to normal fetal state were AC, ALTV and UC. Combining the results of data visualization and Spearman correlation analysis, the CTG features associated with fetal status are listed as follows:
MSTV, AC, MLTV, ASTV, ALTV, UC, LB, Min, FM, DP, Mode, Mean, and Median. In detail, visual analysis and Spearman's correlation analysis found that as the value of heart rate acceleration (AC) increased, the probability of normal state increased, whereas that of suspicious and pathological state reversed. It can be learned that the heart rate acceleration is an important factor to reflect fetal state and accompanies fetal movement to a certain extent. In addition, data visualization and Spearman correlation analysis found that as the percentage of time with abnormal short-term variability (ASTV) increased, the probability of pathology increased and that of the normal gradually decreased.

Forward-stepwise-selection ARA discovered eight essential features and two discriminant rules about assessing normal and pathological fetal status, which verified and complemented the CTG interpretation rules in fetal monitoring guidelines. The results imply that MSTV, AC, Mean, ASTV and Min are the decisive features of normal fetal status, and the main features of pathological fetal status are DP, DS, Variance, and Mean. From data mining perspective, the outcomes of forward-stepwiseselection ARA further validate what the proposed data visualization and Spearman correlation analysis found.

Combining the above analysis with clinical knowledge of fetal monitoring, we obtain 17 features to establish measurement models and structural equation models for analyzing the interpretable mechanisms of evaluating fetal status. The results of measurement models showed that the significant features of 16 passed the hypothesis test and were grouped into five latent variables, namely ACat, BCat, VCat, DCat and UCat. Consequently, from the outcomes of the structural equation model, it can be observed that ACat directly affects fetal status with the standardized path coefficient -0.665 , which reveals that the fetal status is more likely to be identified as normal with the value of ACat increases. In addition, VCat and UCat influence fetal status by affecting BCat and DCat, respectively.

In summary, our study obtained important CTG features for interpreting fetal status and the mechanistic pathways. It reveals that heart rate acceleration and ASTV are key factors that affect fetal status. The international medical guidelines report that the normal state should satisfy that acceleration increases at least $15 \mathrm{sec}-$ onds when the baseline is higher than $15 \mathrm{bpm}$ and continues at least twice in 15 minutes [31, 32]. Street et al. found that STV was strongly associated with metabolic acidosis and dead fetus in the uterus when without high variability [33]. In addition, BCat and DCat are mediating factors that affect fetal status through VCat and UCat, respectively. These findings were verified by the clinical knowledge of prenatal fetal monitoring: Uterine 
Chi/DF=38.737

$\mathrm{GFI}=0.618$

AGFI $=0.487$

RMSEA $=0.217$

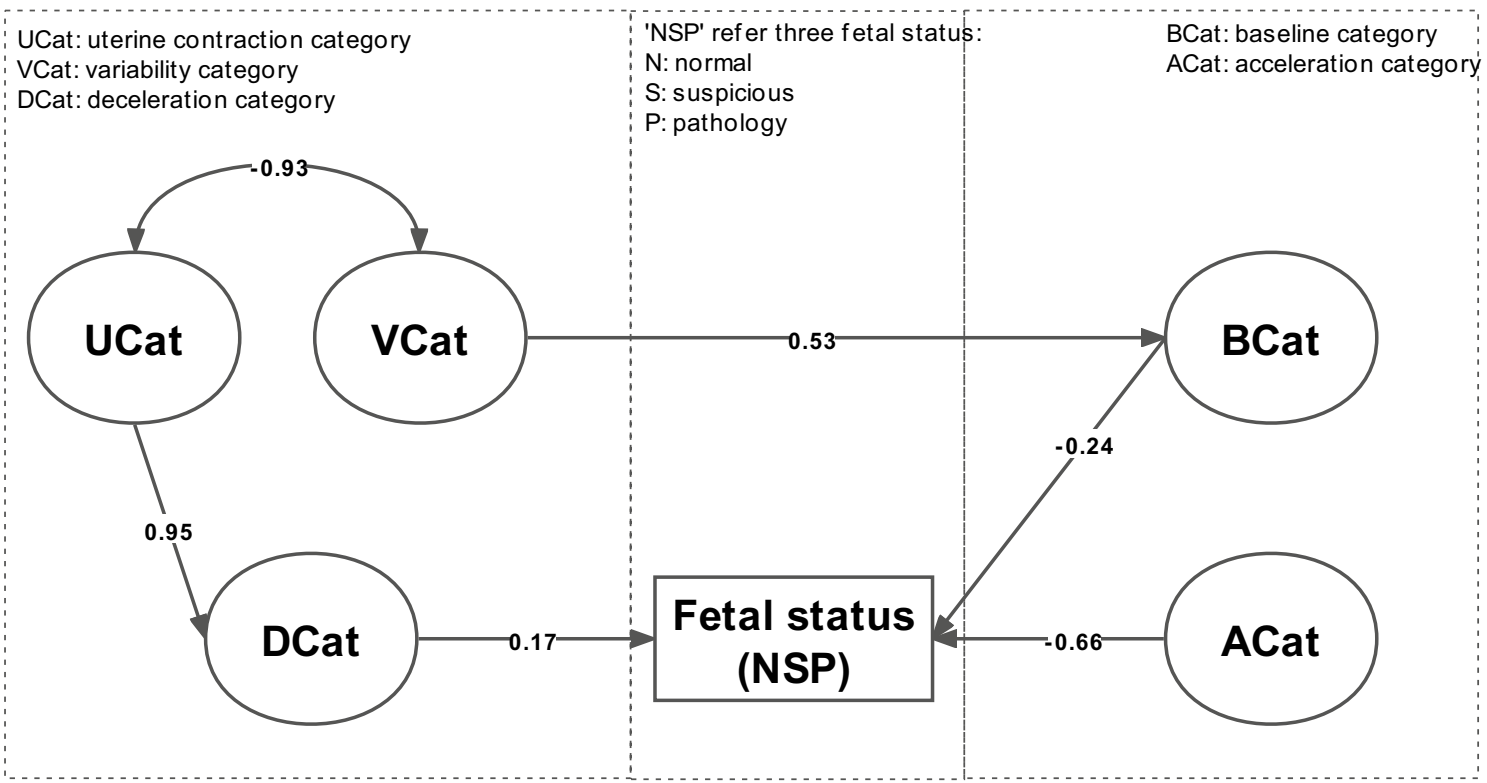

Fig. 6 The structural model for analyzing main factors affecting fetal status evaluation and their path ways. Note: The value of standard path coefficient is indicated by an arrow on one end along with a number; the value of correlation coefficient between Vcat and Ucat is represented by double arrow with a number

contraction blocks the blood flow between the uterus placenta and the decrease of fetal oxygen supply leads to a slow fetal heart rate [34]; Baseline was adjusted by the VCat in SisPorto [13]. These causal relationships between the latent variables and fetal status are beneficial to evidence-based medical research and provide an interpretable reference for fetal status assessment in prenatal fetal monitoring.

\section{Conclusion}

This study analyzed the important CTG features related to the fetal status, explored association rules to supplement fetal monitoring guidelines and causal relationships among latent variables to fetal status using CTG records in different perspectives. Visual analysis was carried out from the perspective of data distribution. Spearman correlation analysis identified important CTG features of great impacts on fetal status. Forwardstepwise-selection ARA excavated the important features and interpretation rules to judge the fetal status as normal and pathological, which verified and supplemented the fetal monitoring guidelines. Structural equation model was used to explore the mechanism of prenatal CTG feature on fetal status, providing an interpretable reference for fetal status assessment in prenatal fetal monitoring.

However, the findings in this study should be interpreted with caution. Firstly, there are various methods for CTG feature computing and analysis, like timedomain and frequency-domain analysis of CTG and FHR recordings. Besides, the same feature or parameter could be defiantly measured by different algorithmic instruments $[7,35]$. Hence, the analysis of CTG records needs to be integrated with other clinical information for a comprehensive interpretation and adequate management. In our study, time-domain morphological features of fetal heart rate and contraction signals measured by the Sisporto program were analyzed. Lastly, except for fetal status assessment, one of the main goals in cardiotocography is the investigation of the linkage between antepartum CTG features and postpartum clinical outcomes [36, 37]. Therefore, our further study will design prospective trials to track the clinical fetal outcome for exploring the relationships between the CTG features and some specific fetal diseases, such as fetal distress, intrauterine growth restriction or neonatal academia. 
Table 6 Standardized path coefficients and $P$ values of the final structural equation model

\begin{tabular}{|c|c|c|c|}
\hline Relationship & Estimate $^{a}$ & C.R. ${ }^{b}$ & $P$ Values ${ }^{c}$ \\
\hline Variance $\longleftarrow$ VCat & -0.700 & -15.527 & $* * *$ \\
\hline MSTV «VCat & -0.886 & -18.369 & $* * *$ \\
\hline ALTV $\longleftarrow$ VCat & 0.608 & & \\
\hline ASTV $\longleftarrow$ VCat & 0.531 & 13.032 & $* * *$ \\
\hline Width $\longleftarrow$ VCat & -0.805 & -17.849 & $* * *$ \\
\hline $\mathrm{LB} \longleftarrow \mathrm{BCat}$ & 0.754 & & \\
\hline Mean $\longleftarrow$ BCat & 0.975 & 30.976 & $* * *$ \\
\hline Median $\longleftarrow$ BCat & 0.992 & 31.908 & $* * *$ \\
\hline Mode $\longleftarrow$ BCat & 0.936 & 29.481 & $* * *$ \\
\hline $\mathrm{DP} \longleftarrow$ DCat & 0.535 & & \\
\hline $\mathrm{DL} \longleftarrow$ DCat & 0.755 & 13.012 & $* * *$ \\
\hline Nzeros $\longleftarrow$ DCat & 0.345 & 7.690 & $* * *$ \\
\hline $\mathrm{AC} \longleftarrow \mathrm{ACat}$ & 0.734 & & \\
\hline Nmax ACat & 0.427 & 7.309 & $* * *$ \\
\hline $\mathrm{FM} \longleftarrow \mathrm{ACat}$ & 0.166 & 3.790 & $* * *$ \\
\hline $\mathrm{UC} \longleftarrow$ UCat & 0.285 & & \\
\hline BCat $\longleftarrow$ VCat & 0.527 & 11.532 & $* * *$ \\
\hline DCat « UCat & 0.952 & 10.599 & $* * *$ \\
\hline UCat $\longleftrightarrow$ VCat & -0.925 & -10.411 & $* * *$ \\
\hline $\mathrm{NSP} \longleftarrow$ ACat & -0.665 & -7.718 & $* * *$ \\
\hline $\mathrm{NSP} \longleftarrow$ BCat & -0.240 & -5.423 & $* * *$ \\
\hline NSP $\longleftarrow$ DCat & 0.173 & 2.856 & 0.004 \\
\hline
\end{tabular}

***Significant at $P<0.001$

${ }^{a}$ Standardized path coefficients of estimate

${ }^{b}$ Critical ratio (C.R.) is the $t$ value of T-test

$c P>0.05$ implied that the hypothetical pathways were false

\section{Abbreviations}

AC: Number of accelerations per second; ACat: Acceleration category; ACOG: American College of Obstetricians and Gynecologists; AdaBoost: Adaptive enhancement; ALTV: Percentage of time with abnormal long term variability; ARA: Association rule analysis; ASTV: Percentage of time with abnormal short term variability; AUC: Area under the curve; BCat: Baseline category; CTG: Cardiotocography; DCat: Deceleration category; DL: Number of light decelerations per second; DP: Number of prolonged decelerations per second; DS: Number of severe decelerations per second; DT: Decision tree; FHR: Fetal heart rate; FIGO: International Federation of Gynaecology and Obstetrics; FM: Number of fetal movements per second; LB: FHR baseline (beats per minute); Max: Maximum of FHR histogram; Mean: Histogram mean; Median: Histogram median; Min: Minimum of FHR histogram; MLTV: Mean value of long term variability; Mode: Histogram mode; NICE: National Institute for Health and Care Excellence; Nmax: Number of histogram peaks; NSP: Normal, suspicious and pathology cases; NST: Non-stress testing; Nzeros: Number of histogram zeros; PCA: Principal component analysis; SEMs: Structural equation models; SOGC: Society of Obstetricians and Gynaecologists of Canada; SVM: Support vector machine; ST: Statistical test; Tendency: Histogram tendency; UC: Number of uterine contractions per second; UCat: Uterine contraction category; Variance: Histogram variance; VCat: Variability category; Width: Width of FHR histogram.

\section{Acknowledgements}

Not applicable.

\section{Authors' contributions}

LH conceptualized and designed the research, performed the data analysis, and drafted the manuscript. ZJ performed the data analysis and drafted the manuscript. RC, QC and JH contributed to the design of the research; $\mathrm{LL}$ validated the experimental results. ZH provided funding acquisition. HW supervised the entire research, reviewed writing and provided funding acquisition. All authors edited the paper and gave final approval for the version to be published. All authors read and approved the final manuscript.

\section{Funding}

This work was funded by Natural Science Foundation of China (Grand No. 61976052) and Medical Scientific Research Foundation of Guangdong Province (Grand No. A2019428). The funding agreements ensured the authors' independence in designing this study, interpreting the data, writing, and publishing the manuscript.

\section{Availability of data and materials}

The data used in this work is publicly available from https://archive.ics.uci.edu/ $\mathrm{ml} /$ datasets/cardiotocography.

\section{Declarations}

\section{Ethics approval and consent to participate}

Not applicable.

\section{Consent for publication}

Not applicable.

\section{Competing interests}

The authors declare that they have no competing interests.

\begin{abstract}
Author details
${ }^{1}$ School of Computer, Guangdong University of Technology, Waihuan West Road, Guangzhou, China. ${ }^{2}$ School of Medical Information Engineering, Guangzhou University of Chinese Medicine, Waihuandong Road, Guangzhou, China. ${ }^{3}$ The First Affiliated Hospital of Jinan University, Tianhe District People's Hospital, Dongpu Road, Guangzhou, China. ${ }^{4}$ Department of Mathematics, College of Science, Shantou University, Daxue Road, Shantou 515063, China. ${ }^{5}$ Guangzhou Sanrui Medical Equipment Co, Gaoke Road, Guangzhou, China.
\end{abstract}

Received: 13 October 2020 Accepted: 6 December 2021

Published online: 20 December 2021

\section{References}

1. Grivell RM, Alfirevic Z, Gyte GM, Devane D. Antenatal cardiotocography for fetal assessment. Cochrane Database Syst Rev. 2015;2015(9):CD007863.

2. Alfirevic Z, Gyte GM, Cuthbert A, Devane D. Continuous cardiotocography (CTG) as a form of electronic fetal monitoring (EFM) for fetal assessment during labour. Cochrane Database Syst Rev. 2017;2(2):CD006066.

3. Cheng Z, Song S. Fetal electronic monitoring. Obstet Gynaecol Reprod Med. 2001;81-92.

4. Haran SS, Everett TR. Antenatal fetal wellbeing. Obstet Gynaecol Reprod Med. 2017;27(2):44-9.

5. SOGC clinical practice guidelines. Guidelines for vaginal birth after previous caesarean birth. Number 155 (Replaces guideline Number 147), February 2005. Int J Gynaecol Obstet 2005;89(3):319-31.

6. Santo S, Ayres-de Campos D, Costa-Santos C, Schnettler W, Ugwumadu A Da Graça LM, et al. Agreement and accuracy using the FIGO, ACOG and NICE cardiotocography interpretation guidelines. Acta Obstet Gynecol Scand. 2017;96(2):166-75.

7. Ayres-de Campos D, Spong CY, Chandraharan E. FIGO consensus guidelines on intrapartum fetal monitoring: cardiotocography. Int J Gynecol Obstet. 2015;131(1):13-24.

8. Chinese Medical Association PMB. Expert consensus on the application of electronic fetal heart monitoring. Chin J Perinat Med 2015;18(007):486-90.

9. Sabiani L, Le Dû R, Loundou A, d'Ercole C, Bretelle F, Boubli L, et al. Intraand interobserver agreement among obstetric experts in court regarding the review of abnormal fetal heart rate tracings and obstetrical management. Am J Obstet Gynecol. 2015;213(6):856.e1. 
10. Dawes GS, Moulden M, Redman CWG. System 8000: computerized antenatal FHR analysis. J Issue. 1991;19(1-2):47-51.

11. Cömert Z, Kocamaz AF. Open-access software for analysis of fetal heart rate signals. Biomed Signal Process Control. 2018;45:98-108.

12. Sbrollini A, Agostinelli A, Burattini L, Morettini M, Di Nardo F, Fioretti S, et al. CTG analyzer: a graphical user interface for cardiotocography. In: 2017 39th annual international conference of the IEEE engineering in medicine and biology society (EMBC). IEEE; 2017. p. 2606-9.

13. Ayres-de Campos D, Bernardes J, Garrido A, Marques-de Sa J, Pereira-Leite L. SisPorto 2.0: a program for automated analysis of cardiotocograms. J Matern-Fetal Med. 2000;9(5):311-8.

14. Bernardes J, Costa-Pereira A. The effect of different sampling intervals on the measurement of intrapartum fetal heart rate variability. Obstet Gynecol. 1997;90(2):318-9.

15. Yang $X$, Fang S, Ling S. Clinical application of fetal heart monitoring for the diagnosis of fetal distress. Mod Med Health. 2017;2017(04):105-7.

16. Sahin $H$, Subasi A. Classification of the cardiotocogram data for anticipation of fetal risks using machine learning techniques. Appl Soft Comput. 2015;33:231-8.

17. Zhang $Y$, Zhao Z. Fetal state assessment based on cardiotocography parameters using PCA and AdaBoost. In: 2017 10th international congress on image and signal processing. BioMedical engineering and informatics (CISP-BMEI). IEEE; 2017. p. 1-6.

18. Nagendra V, Gude H, Sampath D, Corns S, Long S. Evaluation of support vector machines and random forest classifiers in a real-time fetal monitoring system based on cardiotocography data. In: 2017 IEEE conference on computational intelligence in bioinformatics and computational biology (CIBCB). IEEE; 2017. p. 1-6.

19. Zhao Z, Zhang Y, Deng Y. A comprehensive feature analysis of the fetal heart rate signal for the intelligent assessment of fetal state. J Clin Med. 2018:7(8):223

20. Asuncion A, Newman D. UCI machine learning repository. 2007.

21. Huang Z, Zhou Z, He T, et al. Improved classification algorithm for multiclass imbalanced data association. Pattern Recogn Artif Intell. 2015;000(010):922-9.

22. Long L, Peng L, Kejia Z, Shan H, Qian L. Research and application of the improved a priori algorithm. Comput Digit Eng. 2019;47(6):1293-7.

23. Alsolami F, Amin T, Moshkov M, Zielosko B. Comparison of heuristics for optimization of association rules. Fund Inform. 2019;166(1):1-14.

24. Cheng W, Cheng Z. Fetal electronic monitoring. People's Medical Publishing House; 2018.

25. Zhang H, Wang L, Wang J, Hei J, Ruan C. Premature rupture of the fetal membrane combined with subclinical chorioamnionitis negatively affects pregnancy outcomes by a mechanism associated with reduced levels of matrix metalloproteinase-2. Exp Ther Med. 2015;10(2):561-6.

26. Yang H, Li X, Wang ea Z. Expert consensus on electronic fetal heart monitoring application. Chin J Perin Med. 2015;18(7):486-90.

27. Byrne BM. Structural equation modeling with AMOS, EQS, and LISREL: comparative approaches to testing for the factorial validity of a measuring instrument. Int J Test. 2001;1 (1):55-86.

28. Wen Z, Hau KT, Herbert WM. Structural equation model testing: cutoff criteria for goodness of fit indices and chi-square test. Acta Psychol Sin. 2004:36(02):186-94

29. Hernán MA, Robins JM. Causal inference. CRC; 2010

30. Koller D, Friedman N. Probabilistic graphical models: principles and techniques. MIT Press; 2009.

31. Tomáš P, Krohova J, Dohnalek P, Gajdoš P. Classification of cardiotocography records by random forest. In: 2013 36th international conference on telecommunications and signal processing (TSP). IEEE; 2013. p. 620-923.

32. Shah SAA, AzizW, Arif M, Nadeem MSA. Decision trees based classification of cardiotocograms using bagging approach. In: 2015 13th international conference on frontiers of information technology (FIT). IEEE; 2015. p. 12-7.

33. Street P, Dawes G, Moulden M, Redman C. Short-term variation in abnormal antenatal fetal heart rate records. Am J Obstet Gynecol. 1991;165(3):515-23.

34. Elements of causal inference: foundations and learning algorithms. Cambridge; 2017.

35. Spencer JA. Clinical overview of cardiotocography. BJOG Int J Obst Gynaecol. 1993;100:4-7.
36. Agostinelli A, Palmieri F, Biagini A, Sbrollini A, Burattini L, Di Nardo F, et al. Relationship between deceleration areas in the second stage of labor and neonatal acidemia. In: 2016 computing in cardiology conference (CinC). IEEE; 2016. p. 897-900.

37. Ferrario M, Aletti F, Baselli G, Signorini MG, Cerutti S. Heart rate variability analysis for the monitoring of fetal distress and neonatal critical care. Heart rate variability (HRV) signal analysis clinical applications. Press; 2012 p. 24.

\section{Publisher's Note}

Springer Nature remains neutral with regard to jurisdictional claims in published maps and institutional affiliations.

Ready to submit your research? Choose BMC and benefit from

- fast, convenient online submission

- thorough peer review by experienced researchers in your field

- rapid publication on acceptance

- support for research data, including large and complex data types

- gold Open Access which fosters wider collaboration and increased citations

- maximum visibility for your research: over $100 \mathrm{M}$ website views per year

At BMC, research is always in progress.

Learn more biomedcentral.com/submissions 